\title{
Anaesthetic Management of a Patient During Transurethral Resection of Prostate with Iatrogenic Intraperitoneal Bladder Explosion: An Atypical Event
}

\author{
Ritwiza Thakur', Smriti Anand2*, Rakesh Sadhu ${ }^{3}$
}

\begin{abstract}
1Postgraduate Student, Department of Ananesthesia, Maharishi Markandeshwar Medical College and Hospital, Kumarhatti, Solan, Himachal Pradesh, India. Email ID: drritwizathakur@gmail.com

Orcid ID: 0000-0002-0909-1418.

2Professor, Department of Ananesthesia, Maharishi Markandeshwar Medical College and Hospital, Kumarhatti, Solan, Himachal Pradesh, India.

Email ID: gupta.smriti1@gmail.com

Orcid ID: 0000-0001-5788-6285

3Professor and Head, Department of Ananesthesia, Maharishi Markandeshwar Medical College and Hospital, Kumarhatti, Solan, Himachal Pradesh, India. Email ID: rsadhu2008@gmail.com

Orcid ID: 0000-0002-0287-2203
\end{abstract}

*Corresponding author

Received: 02 November 2021

Revised: 14 December 2021

Accepted: 23 December 2021

Published: 18 February 2022

\begin{abstract}
Transuretheral resection of prostate (TURP) is one of the most commonly performed urological procedure. Though considered to be safe, it is sometimes associated with atypical complications. We hereby report a successful anaesthetic management of bladder explosion, a very rare complication of TURP, occuring towards the end of the procedure at the time of removal of resectoscope. The prompt recognition and management of the same led to uneventful postoperative course.
\end{abstract}

Keywords:-Transuretheral resection of prostate, Benign prostatic hyperplasia, Bladder explosion

\section{INTRODUCTION}

Transurethral resection of prostate (TURP) is one of the most commonly performed urological procedures for bladder outlet obstruction resulting from benign prostatic hypertrophy $(\mathrm{BPH})$ and involves the endoscopic resection of an enlarged prostate; its other indications being obstructive nephropathy, bladder calculus, prostatic abscess as well as obstructive azoospermia to open ejaculatory ducts. Even though the surgery is generally believed to be safe, lifethreatening atypical complications can occur.Bladder perforation in the form of bladder explosion after transurethral prostate resection is one such unique and rare event occurring in $0.01 \%-0.02 \%$ of cases characterized by a high and sudden increase in intravesical pressure.[1] Cassuto reported the first incidence of bladder explosion in 1926.There have been less than 20 cases documented in the literature and most of them were treated by a laparotomy.[2,3] Small 
Annals of International Medical and Dental Research E-ISSN: 2395-2822 | P-ISSN: 2395-2814

Vol-8, Issue-2 | March-April 2022

DOI: 10.53339/aimdr.2022.8.2.4

Page no- 15-18 | Section- Research Article (Ananesthesia)

bubbles, such as hydrogen, oxygen, and other gases formed by tissue and water combustion, are frequently seen on an arc of the resectoscope.The contact of the electrocautery with a collection of gases created during the resection technique is believed to be the cause of bladder explosion during transurethral resection of prostate. (TURP).The injury can range from a minor tear to a massive rupture. We present the case of a 59-year-old man, ASA II with benign prostatic hyperplasia (BPH) who had a bladder explosion during TURP, towards the end of procedure, which was timely detected and patient underwent laparotomy to repair the defect. The patient was managed propitiously and discharged.

\section{CASE REPORT}

59 yrs. old male weighing $75 \mathrm{~kg}$ presented in surgery OPD with complaints of intermittent haematuria, refractory urinary retention for 4 months and was on continuous per urethral catheterisation. Patient was diagnosed with grade III $\mathrm{BPH}$ and was posted to undergo transurethral resection of prostate (TURP) surgery under spinal anaesthesia. In the preanesthetic workup - detailed history was taken and examination done. He had no comorbidities. He had history of head injury 5 yrs back and managed conservatively. Patient was reformed smoker. His physical examination \&systemic examination were normal. On checking his vitals SpO2 was 98$100 \%$ on room air, pulse rate (PR)- 72 bpm, respiratory rate (RR)-16pm and blood pressure (BP)was 148/90 mm Hg. Airway examination was normal. Mets was > 4.Baseline investigations were done which were found to be normal. 12-lead ECG done showed Right bundle branch block with ST depression in
V1,V2,V5,V6. Cardiologist opinion was sought. 2D ECHO was done for further evaluation. $\mathrm{ECHO}$ finding revealed concentric left ventricular hypertrophy, grade 1 diastolic dysfunction, normal left ventricular systolic function. The Patient was taken up for surgery under ASA II and subarachnoid block (SAB) planned. Patient was premedicated with Alprazolam0.5mg night prior to surgery and prophylactic antibiotic was administered in the preoperative ward. In Operation theatre standard monitors were applied and baseline vitals noted. Large bore iv cannula was secured and patient was preloaded with $500 \mathrm{ml}$ of Ringer lactate. SAB was given under all aseptic precautions with $15 \mathrm{mg}$ of hyperbaric bupivacaine. Adequate block achieved and surgery proceeded.The hemodynamic parameters were monitored throughout the procedure.

TURP was performed with monopolar resectoscope, using $1.5 \%$ Glycine. The operating time for $90 \mathrm{~g}$ prostate was around 80 minutes. A loud thud explosion sound was heard near the end of the procedure, at the time of removal of resectscope when the resection was complete. The surgeon performed bladder irrigation but blood tinged fluid returned. Patient complained of severe pain and thereafter became restless and confused. The blood pressure abruptly dropped to- $80 / 60 \mathrm{mmHg}$ followed by severe bradycardia (HR-32-38 beats per minute),injection atropine $0.6 \mathrm{mg}$ intravenous had to be repeated twice followed by $6 \mathrm{mg}$ boluses of injection mephenteramine, however the blood pressure did not rise for which Injection Nor Adrenaline was started at the rate of $2 \mu \mathrm{g} /$ minute. A rupture of the 
Annals of International Medical and Dental Research E-ISSN: 2395-2822 | P-ISSN: 2395-2814

Vol-8, Issue-2 | March-April 2022

DOI: 10.53339/aimdr.2022.8.2.4

Page no- 15-18 | Section- Research Article (Ananesthesia)

intraperitoneal bladder was suspected, irrigation was promptly discontinued and decision for exploratory laparotomy taken by the operating surgeon. General anaesthesia was induced with injection propofol, succinylcholine $75 \mathrm{mg}$ given to facilitate endotracheal intubation and anaesthesia was maintained with isoflurane, and combination of oxygen and nitrous oxide and intermittent vecuronium. The abdomen was explored by a lower midline incision.On exploration, a 6-7 $\mathrm{cm}$ defect was noticed in the dome of the urinary bladder. There was no associated gastrointestinal or vascular damage. Homeostasis was achieved and bladder defect closed in two layers. A suprapubic catheter was placed. Patient was shifted to ICU with inotropic support and injection non adrenaline was tapered gradually with the improvement of blood pressure. The postoperative course was uneventful.

\section{DISCUSSION}

Despite recent and ongoing advancements in surgical and nonsurgical treatment options for prostate hyperplasia, TURP remains one of the most common and reasonably safe procedures performed worldwide. However, major peri and postoperative complications can emerge, necessitating a comprehensive and systematic approach to their management .Bladder perforation during TURP is an uncommon complication that mainly arises from mechanical injury caused by the resectoscope or by an increase in intravesical pressure, which can lead to intravesical explosion, an extremely rare complication. It occurs when a combination of explosive gases builds up.Electrocautery generates a variety of gases, including hydrogen, carbon monoxide, and acetylene. However, whether these are caused by electrolysis or thermolytic destruction of tissue is not known.With similar results, Ning et al. analyzed the gases formed in human urinary bladders following transurethral surgery.[4] He believed that intracellular water electrolysis produces hydrogen.The mixture is not explosive because the oxygen level is so low. However, when more atmospheric air (containing $21 \%$ oxygen) enters the bladder, cautery triggers an explosion. According to von Dittel, bladder overdistension with water can result in either intraperitoneal or extraperitoneal bladder rupture, but gasinduced bladder rupture is usually always intraperitoneal.[5] When the resectoscope is opened for any reason, or when irrigation fluid bottles are replaced, air can be introduced into the bladder through leakage into the irrigation tubing. As a result, variables that control air entrance into the bladder should be carefully sought, such as continuous irrigation, airtight irrigation system connections, and meticulous manipulation of the evacuator bulb. In particular, the surgeon's attention is required to assess the presence of small air bubbles that may form in the bladder during the TURP, and in such cases, it is recommended that, if possible, the prostatic resection be avoided, especially in cases of benign prostatic hypertrophy, to reduce the time of surgical procedures and gas production. In many cases, bladder explosion occurred near the end of TURP procedures for prostate cancer, which lasted between 75 and 80 minutes.[6] The bladder explosion in this case occurred towards the end of the surgery while the surgeon removed the resectoscope. There are various strategies which when judiciously employed can avert this atypical complication 
Annals of International Medical and Dental Research

E-ISSN: 2395-2822 | P-ISSN: 2395-2814

Vol-8, Issue-2 | March-April 2022

DOI: 10.53339/aimdr.2022.8.2.4

Page no- 15-18 | Section- Research Article (Ananesthesia)

which include: application of subtle suprapubic pressure to facilitate the withdrawl of gases from the bladder dome while emptying the bladder, use of suprapubic catheters for efficient bladder clearing, avoiding the entry of oxygen into the bladder through leaky connections, employing low power electocautery settings, use of continuous flow resectscopes and directing the angle of the beak of the resectscope towards the dome while emptying the bladder.[7]

The anaesthesiologist may communicate with the urologist regarding these points to avert this rare catastrophe.

\section{REFERENCES}

1. Gupta J, Gupta A. Intraperitoneal bladder explosion during transurethral surgery. BMJ Case Rep. 2019;12(7):e229580. doi: 10.1136/bcr-2019-229580.

2. OğuzG, Subaşı D, Kaya M, Güven $O$, Ünver S. Intravesical explosion: a rare complication of transurethral resection of prostate. J Anesth. 2013;27(1):145-6. doi: 10.1007/s00540-012-1474-7.

3. Hambleton BF, Lackey RW, Van Duzen RE. Explosive gases formed during electrotransurethral resections. JAMA. 1935;105(9): 645-646.

4. Ning TC Jr, Atkins DM, Murphy RC. Bladder explosions during transurethral surgery. J Urol. 1975;114(4):536-9. doi: 10.1016/s0022-5347(17)670770 .

\section{CONCLUSIONS}

Transuretheral resection of prostate (TURP) though considered to be safe procedure may sometimes be associated with certain atypical complications. One such rare event is the bladder perforation in the form of bladder explosion. However the prompt recognition and management of the same can help to avert any untoward event.

5. Gupta J, Gupta A. Intraperitoneal bladder explosion during transurethral surgery. BMJ Case Rep. 2019;12(7):e229580.. doi:10.1136/bcr-2019-229580

6. Seitz M, Soljanik I, Stanislaus P, Sroka R, Stief C. Explosive gas formation during transurethral resection of the prostate (TURP). Eur J Med Res. 2008;13(8):399-400.

7. Agrawal A, Darakh P, Patil M, Mahajan A. Intravesical explosion in a bladder diverticulum during transurethral resection of prostate. Int J Contemp Med Res. 2018;5(7):G6-G7.

Source of Support: Nil, Conflict of Interest: None declared 